\title{
Mechanisms of Biotherapy Effect of Shenfoweikang Herbs on Gastric Carcinoma Cells
}

\author{
Xiao-ping WANG ${ }^{1, a,}$, , Huan-ping $\mathrm{LIN}^{1}$, Qiao-xia WANG ${ }^{2}$, Bing $X \mathrm{U}^{1}$, \\ Xuan QU ${ }^{1}$, Bao-ning $\mathrm{QI}^{1}$ and $\mathrm{Na} \mathrm{CHANG}^{1}$ \\ ${ }^{1}$ Key Laboratory of Molecular Biology and Pathology, Shaanxi University of Chinese \\ Medicine, Xianyang, Shaanxi 712046, PR China \\ ${ }^{2}$ Departments of Infectious Diseases, Xi'an Central Hospital, Xi'an, Shaanxi \\ 710000, PR China \\ awxphd@aliyun.com \\ ${ }^{*}$ Corresponding author
}

Keywords: Biomaterial, Biotherapy, Mechanism, Gastric carcinoma.

\begin{abstract}
To explore the biotherapy effect of Shenfoweikang herbs in treatment of gastric cancer, BALB/C mice were grafted with a mouse gastric adenocarcinoma cell line MFC as the experimental model. The mice were divided into four groups. Mice in the experimental groups received different doses of Shenfoweikang herbs over a 60-day period starting at the first day after grafting. Mice received saline as controls. All the mice were sacrificed at 61 days after being grafted. Tumor size was periodically measured during the life of the mice and tumor weight was determined by an electron balance immediately after the animals killed. Serum cytokines, granzyme $\mathrm{B}$ and perforin were examined by the ELISA method. The anti-tumor effect was detected by the cytotoxic T-lymphocyte (CTL) assays. Our results demonstrated that Shenfoweikang herbs could inhibit the growth of gastric cancer by activating the CTLs and inducing the secretion of cytokines, perforin and granzyme B. Our study suggests that Shenfoweikang herbs inhibited the proliferation of gastric carcinoma by activating the effects of immune cells, which may lay a better basis for further study on gastric cancer biotherapy.
\end{abstract}

\section{Introduction}

In clinic studies, Chinese Shenfoweikang herbs had been found to have effect on pre-malignant lesion, especially on gastric diseases [1, 2]. The Shenfoweikang decoction might inhibit gastric carcinoma cell proliferation and cause tumor cell death. Apoptosis plays a crucial role in the proliferation and turnover of cells in various tumors. It has been clear that its extent is often enhanced in tumor by many anticancer drugs, such as cytotoxic drugs, hormone, or some Chinese herbal medicine [3-5]. Researches indicated that Chinese herbs could enhance apoptosis of human gastric cancer grafted in mice [5-7].

In the present study, we investigated whether the Shenfoweikang herbs could induce the effects of the immune cells against gastric cancer grafted onto mice, further confirming the anti-tumor mechanism of the Chinese Shenfoweikang herbs.

\section{Material and Methods}

\section{Mice and Tumor Cell Lines}

Six- to eight-week-old female BALB/C mice were purchased from the Experimental 
Animal Center at Fourth Military Medical University. All animals were maintained under specific-pathogen-free conditions, and all procedures were performed according to approved protocols and in accordance with recommendations for the proper care of laboratory animals. The investigation was approved by the Ethics Committee on animal Study at Shaanxi University of Chinese Medicine (2004-4B).

Drugs. The Shenfoweikang decoction consists of Codonopsis pilosula (Franch) Nannf., Atractylodes macrocephala koidz, Poria cocos (Schw.) Wolf, Glycyrrhiza uralensis Fisch. The concentration of the Shenfoweikang decoction was $240 \mathrm{~g} / \mathrm{L}$.

Administration. The mice were randomly divided into 4 groups, one control and the other three experimental groups, which are assigned to receive the Shenfoweikang decoction. Each animal in the three experimental groups was given $2.0 \mathrm{~mL}, 1.0 \mathrm{~mL}$ and $0.5 \mathrm{~mL}$ of the Shenfoweikang decoction through gastric perfusion every day over a 60 -day period beginning at 1 st day after grafted. The control group only received normal saline according to the same schedule. All the mice were killed at 61 st day after grafted.

Perforin and Granzyme B ELISA Assay. The ELISA was used to measure the cytokine perforin and granzyme B in serum collected from above mice according to manufacturer's instruction. The OD values were obtained using an ELISA Reader System.

Cytotoxic T-lymphocyte (CTL) Assays. BALB/C mice were administrated as described above. The 61 day, $2.5 \times 10^{7}$ splenocytes were collected from the different mice groups and cultured with 10 units/ml of mouse interleukin (IL)-2 in RPMI 1640 supplemented with $10 \% \mathrm{FCS}$ at $37{ }^{\circ} \mathrm{C}$ in $5 \% \mathrm{CO}_{2}$. After 5 days of stimulation, the viable splenocytes were recovered and used as effector cells, and the MFC cells were used as target cells. The Non-Radioactive Cytotoxicity Lactate Dehydrogenase (LDH) release assay Kit (Promega, 249 USA) was performed to measure the effector cells against MFC tumor cells in the ratios of 10:1, 20:1 and 40:1, according to the manufacturer's protocol. Specific lysis was calculated according to the formula: percent specific lysis $=[$ (experimental release value - effector spontaneous release value - target spontaneous release value)/(target maximum release value - target spontaneous release value) $] \times 100$. Results shown are representative of experiments repeated three times.

In Vivo Tumor Therapeutic Experiments. To confirm whether Shenfoweikang herbs inhibited the growth of established tumors, one control and the other three experimental groups were assigned to receive the Shenfoweikang decoction. Each animal in the three experimental groups was given $2.0 \mathrm{~mL}, 1.0 \mathrm{~mL}$ and $0.5 \mathrm{~mL}$ of the Shenfoweikang decoction by gastric perfusion every day over a 60-day period beginning at 1 st day after grafting. The control animals received normal saline according to the same schedule. $5 \times 10^{6}$ MFC tumor cells were washed after enzymatic digestion and resuspended in $0.2 \mathrm{ml}$ of PBS per animal, then injected s.c. into the left flank. Tumor size was measured in two dimensions with calipers every 3 days one week after tumor inoculation. Tumor volume was calculated using the formula: $\mathrm{V}=$ $\left(a^{2} b\right) / 2$.

Statistical Analysis. All data expressed as means \pm S.D. The Student's t test was performed to analyze the significance of differences in different groups of mice. $P<0.05$ was considered statistically significant. 


\section{Results}

\section{Inhibition of Tumor Growth by Shenfoweikang Herbs}

Compared with the control group, tumor growth (size and weight) was significantly inhibited by treatment with the Shenfoweikang decoction $(P<0.05$, Table 1$)$. The results showed that the higher the concentration of Shenfoweikang herbs, the less the tumor weight and size. There was a significant difference between the Chinese herbs and control group. The morphological changes of tumor were showed as Figure 1. Compared with the control group, the gastric adenocarcimoma glands were less and smaller in Shenfoweikang decoction treated group.

Table 1 Shenfoweikang herbs induced effects on gastric cancer $(\mathrm{x} \pm \mathrm{s})$

\begin{tabular}{|c|c|c|}
\hline Treatment & Tumor weight $(\mathrm{g})$ & Tumor size $\left(\mathrm{mm}^{3}\right)$ \\
\hline High-dose Shenfoweikang Decoction & $0.48 \pm 0.32^{\mathrm{a}}$ & $257.44 \pm 36.43^{\mathrm{a}}$ \\
\hline Middle-dose Shenfoweikang Decoction & $0.64 \pm 0.25^{\mathrm{b}}$ & $334.32 \pm 32.42^{\mathrm{b}}$ \\
\hline Low-dose Shenfoweikang Decoction & $0.82 \pm 0.37^{\mathrm{c}}$ & $452.56 \pm 27.66^{\mathrm{c}}$ \\
\hline Saline & $2.03 \pm 0.26$ & $588.42 \pm 34.23$ \\
\hline
\end{tabular}

${ }^{\mathrm{a}} P<0.05,{ }^{\mathrm{b}} P<0.05,{ }^{\mathrm{c}} P<0.05$ vs control group.
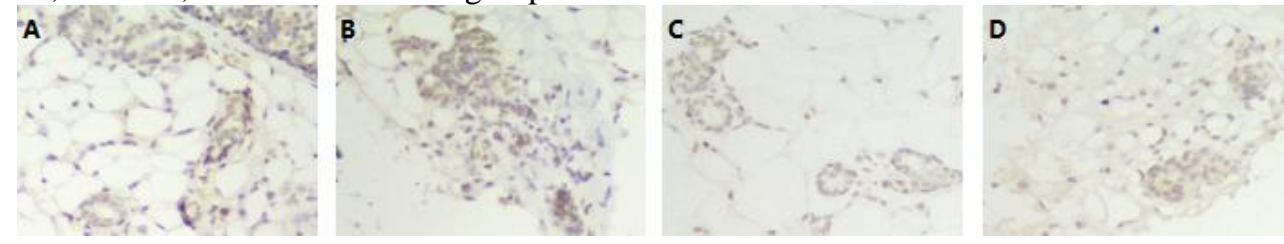

Figure 1. The morphological changes of gastric adenocarcimoma in different groups, $\times 200$

A.Control group, B. Low-dose group, C. Middle-dose group, D. High-dose group.

Shenfoweikang Herbs Induced the Up-regulation of Perforin and Granzyme B in Vivo. The concentrations of perforin and granzyme $B$ in serum from the experimental mice were much more higher compared with those from mice treated with PBS $(P<0.05$, Table 2). The results indicated that Shenfoweikang herbs could induce significant immune $\mathrm{T}$ cells response compared with the control group.

Table 2 Immunological parameters of mice immunized with therapeutic peptide vaccines $(\mathrm{x} \pm \mathrm{s})$

\begin{tabular}{|c|c|c|}
\hline Treatment & Perforin $(\mu \mathrm{g} / \mathrm{ml})$ & Granzyme B $(\mu \mathrm{g} / \mathrm{ml})$ \\
\hline High-dose Shenfoweikang Decoction & $386.24 \pm 16.36^{\mathrm{a}}$ & $315.58 \pm 12.64^{\mathrm{a}}$ \\
\hline Middle-dose Shenfoweikang Decoction & $277.83 \pm 13.34^{\mathrm{b}}$ & $251.44 \pm 10.72^{\mathrm{b}}$ \\
\hline Low-dose Shenfoweikang Decoction & $156.24 \pm 11.48^{\mathrm{c}}$ & $135.76 \pm 7.94^{\mathrm{c}}$ \\
\hline Saline & $6.78 \pm 1.49$ & $5.79 \pm 2.65$ \\
\hline
\end{tabular}

${ }^{\mathrm{a}} P<0.05,{ }^{\mathrm{b}} P<0.05,{ }^{\mathrm{c}} P<0.05$ vs control group.

Shenfoweikang Herbs Elicited CTLs Response. Cytotoxicity assay showed that splenocytes cells from mice treated with Shenfoweikang herbs exhibited higher cytolytic effects on MFC target cells than those from mice vaccinated with PBS $(P<$ 0.05 , Table 3). In contrast, splenocytes from the PBS control showed a weaker ability of target cells lysis. Our results indicated that Shenfoweikang herbs were effective inducers for immune cell activity.

Table 3 Shenfoweikang herbs elicited cytolytic effects on gastric cancer $(\mathrm{x} \pm \mathrm{s})$

\begin{tabular}{|c|c|c|}
\hline \multirow{2}{*}{ Treatment } & \multicolumn{2}{|c|}{ Inhibition Rate (\%) } \\
\cline { 2 - 3 } & Splenocytes : MFC cells (10:1) & Splenocytes : MFC cells (40:1) \\
\hline High-dose Shenfoweikang Decoction & $36.62 \pm 7.86^{\mathrm{a}}$ & $68.53 \pm 10.83^{\mathrm{a}}$ \\
\hline Middle-dose Shenfoweikang Decoction & $22.84 \pm 8.43^{\mathrm{b}}$ & $43.25 \pm 8.73^{\mathrm{b}}$ \\
\hline Low-dose Shenfoweikang Decoction & $15.72 \pm 7.68^{\mathrm{c}}$ & $24.78 \pm 7.56^{\mathrm{c}}$ \\
\hline Saline & $6.78 \pm 1.49$ & $5.79 \pm 2.65$ \\
\hline
\end{tabular}

${ }^{\mathrm{a}} P<0.05,{ }^{\mathrm{b}} P<0.05,{ }^{\mathrm{c}} P<0.05$ vs control group. 


\section{Discussion}

Gastric carcinoma is one of the most common malignant gastrointestinal carcinoma in the world. At present gastric carcinoma is still detected later in most patients throughout the world, and even with curative resection, they remain at a high risk of relapse and mortality. Thus, there is a great need for effective adjuvant therapy for patients with gastric carcinoma. Our previous clinic studies suggested that Chinese herbal recipe Shenfoweikang have therapeutic effects on gastric pre-malignant lesion, with increasing the reversal of the atrophic gastritis, decreasing the recurrence and improving the life quality $[1,2]$. Because of its lower toxic side-effect compared with chemical therapy, it is worth to make a further research on its anti-cancer mechanism.

Similar to the other malignant tumors, gastric carcinoma is always accompanying with abnormal cell proliferation and differentiation. In the present study, we found that after treated with Shenfoweikang herbs, the growth of tumor were inhibited compared with the control group. We presumed that the herbs could activate the immune cells to attack the tumor cells as well as induce the apoptosis of the tumor cells directly [8-10]. The results showed that Shenfoweikang herbs could activate the immune cells to secret cytokines, perforin and granzyme B, which are able to induce the apoptosis and necrosis of tumor cells. We also found that splenocytes were activated and elicited the definite cytotoxic effects on tumor cells, which were likely to secret cytokines, such as perforin and granzyme B to exert their immune effects. It has been verified that immune cells could induce the apoptosis of tumor cells [11]. Apoptosis is a complex, tightly regulated, and active cellular process by which individual cells are triggered to undergo programmed cell death, and simultaneously will not injury neighboring cells or elicit any inflammatory reactions $[11,12]$. Various triggering factor initiate corresponding proteo-lysis cascade reaction depending on mitochondrion or APO-1/FAS/CD95 receptor mediate apoptotic pathways [12, 13]. There are many oncogenes and tumor suppressor gene products in the regulation and execution of apoptosis. The results suggest that the mechanism of the inhibition of gastric cancer cells in vivo by Shenfoweikang herbs is related with activating immune cells and further inducing apoptosis.

\section{Conclusions}

Shenfoweikang herbs inhibited gastric cancer cell growth. The anti-tumor effect of Shenfoweikang herbs lies in activating immune cells to secret certain cytokines, such as perforin and granzyme B to inhibit and kill tumor cells. The detailed molecular mechanism of Shenfoweikang herbs inhibiting gastric cancer cells still needs further investigation.

\section{Acknowledgements}

This work is supported by the Scientific Research Program of Shaanxi Provincial Education Department (No.2007JK233, 2010JK484, 14JS025), Shaanxi Administration of Traditional Chinese Medicine (No.15-SCJH001, JCPT001) and Natural Science Basic Research Plan in Shaanxi Province of China (No.2016JM8023, 2016JM8150).

\section{References}

[1] D.F. Schafer, M.F. Sorrell, Hepatocellular carcinoma, Lancet, 353 (1993) 1253-1257. 
[2] Z.Y. Tang, Hepatocellular carcinoma--cause, treatment and metastasis, World J. Gastroenterol. 7 (2001) 445-454.

[3] R. Wong, C. Frenette, Updates in the management of hepatocellular carcinoma, Gastroenterol. Hepatol. (NY). 7 (2011) 16-24.

[4] P. Hanke, C. Rabe, M. Serwe, S. Böhm, C. Pagenstecher, T. Sauerbruch, W.H. Caselmann, Cirrhotic patients with or without hepatocellular carcinoma harbour AFP-specific T-lymphocytes that can be activated in vitro by human alpha-fetoprotein, Scand. J. Gastroenterol. 37 (2002) 949-955.

[5] J.A. Marrero, K.S. Henley, The role of serum biomarkers in hepatocellular carcinoma surveillance, Gastroenterol. Hepatol. (N Y). 7 (2011) 821-823.

[6] J.H. Zhong, H. Li, L.Q. Li, X.M. You, Y. Zhang, Y.N. Zhao, J.Y. Liu, B.D. Xiang, G.B. Wu, Adjuvant therapy options following curative treatment of hepatocellular carcinoma: a systematic review of randomized trials, Eur. J. Surg. Oncol. 38 (2012) 286-295.

[7] H.P. Lin, S.C. Li, Protective effects of Shenfoweikang on acute gastric mucous jnjury on rat, Shaanxi Journal of Traditional Chinese Medicine 28 (2007) 1422-1423. (In Chinese)

[8] S.C. Li, F. Wang, H.P. Lin, X.Y. Zhou, Effects of Shenfoweikang on the secretion of serum gastrin and motilin in chronic atrophic gastritis, Jiangxi Journal of Traditional Chinese Medicine 38 (2007) 68-69. (In Chinese)

[9] A.G. Zhao, H.L. Zhao, X.J. Jin, J.K. Yang, L. Tang, Effects of Chinese Jianpi herbs on cell apoptosis and related gene expression in human gastric cancer grafted onto nude mice, World J. Gastroenterol. 8 (2002) 792-796.

[10] J. Li, G.Z. Sun, H.S. Lin, Y.X. Pei, X. Qi, C. An, The herb medicine formula "Yang Wei Kang Liu" improves the survival of late stage gastric cancer patients and induces the apoptosis of human gastric cancer cell line through Fas/Fas ligand and Bax/Bcl-2 pathways, Int. Immunopharmacol. 8 (2008) 1196-1206.

[11] A. Ashkenazi, V.M. Dixit, Death Receptors: Signaling and Modulation, Science 281 (1998) 1305-1308.

[12] D.R. Green, Apoptotic pathways: the roads to ruin, Cell 94 (1998) 695-698.

[13] M. Muller, S. Wilder, D. Bannasch, D. Israeli, K. Lehlbach, L.W. Min, p53 activates the CD95 (APO-1/Fas) gene in response to DNA damage by anticancer drugs, J. Exp. Med. 188 (1998) 2033-2045. 